\section{Ultrasensitive protein detection in blood serum using gold nanoparticle probes by single molecule spectroscopy}

\author{
Jiji Chen, Chungang Wang, and Joseph Irudayaraj \\ Purdue University, Birck Nanotechnology and Bindley \\ Bioscience Center, 225 South University Street, 215 ABE \\ Building, West Lafayette, Indiana 47907
}

\begin{abstract}
A one-step rapid and ultrasensitive immunoassay capable of detecting proteins in blood serum is developed using gold nanoprobes and fluorescence correlation spectroscopy (FCS). In this approach we take advantage of the inherent photoluminescence property of gold nanoparticles (GNPs) to develop a fluorophore-free assay to observe binding entities by monitoring the diffusion of bound versus unbound molecules in a limited confocal volume. 40-nm GNPs conjugated separately with rabbit anti-lgG (Fc) and goat anti-lgG (Fab) when incubated in blood serum containing $\lg$ forms a sandwich structure constituting dimers and oligomers that can be differentiated by to detect IgG in blood serum at a limit of detection (LOD) of $5 \mathrm{pg} / \mathrm{ml}$. The novelty of integrating GNPs with FCS to develop a sensitive blood immunoassay brings single molecule methods one step closer to the clinic. ๑ 2009 Society of Photo-Optical Instrumentation Engineers. [DOI: 10.1117/1.3183789]
\end{abstract}

Keywords: gold nanoparticles; fluorescence correlation spectroscopy; detection; blood serum.

Paper 08461LR received Dec. 26, 2008; revised manuscript received Mar. 20, 2009; accepted for publication May 26, 2009; published online Jul. 27, 2009

Immunoassays play a prominent role in clinical diagnostics, which are based on specific molecular recognition of antigens by antibodies. Although many advances such as electrochemical biosensors, ${ }^{1}$ surface-enhanced Raman scattering ${ }^{2}$ have enhanced the limit of detection (LOD), the quest for a simpler and more sensitive assay format persists. It is known that gold nanoparticles (GNP) posses an enhanced degree of absorption and scattering due to the surface plasmon oscillation of the electron cloud in metal particles to yield light emission several orders of magnitude compared to some of the conventional dyes. ${ }^{3}$ The strong photon luminescence, light scattering property, and biocompatibility of GNP have been exploited for cell imaging ${ }^{4}$ and photothermal therapy. ${ }^{5}$ Past work on GNP photon luminescence has focused mostly on qualitative application of microscopic imaging and assessment. Our motivation here is to develop a quantitative assay for the very first time in blood serum using fluorescence correlation spectroscopy at a single particle level.

Address all correspondence to: Joseph Irudayaraj, Profesor of Biological Engineering, Purdue University, 225 S. University Street, 215 ABE Building, West Lafayette, IN 47907. Tel: 765-494-0388; E-mail: josephi@purdue.edu
Recently, fluorescence correlation spectroscopy (FCS), a single molecule technique, has been used for single molecule kinetics and biomolecular interaction studies in living cells, ${ }^{6}$ antigen detection, ${ }^{7}$ and freely diffusing gold nanorod in vivo imaging. ${ }^{8}$ Liu et al. demonstrated in a recent study that prostate-specific antigens can be detected by dynamic light scattering (DLS) with a detection limit of $0.5 \mathrm{ng} / \mathrm{mL}$ in neat buffers. ${ }^{9}$ We hypothesize that if GNP could be used as labels instead of fluorophores, then the present FCS technique could be adapted to observe the diffusion mode of not only a single gold nanoparticle but also higher-order aggregates of GNP. Because the diffusion time of GNPs passing through the confocal volume increases on formation of aggregates constituting oligomers, by studying the diffusion properties of gold particle-based oligomers from autocorrelation analysis, a highly sensitive FCS-based immunoassay could be developed. In addition to that, we also show that diffusion and hydrodynamic diameter of GNP and their aggregates could be consistently monitored in blood serum for quantification.

We report on the development of a one-step assay to detect human IgG as an antigen protein spiked in 20\% dog blood serum using GNP conjugated with different antihuman IgG antibody fragments to result in the formation of oligomers of different orders. An example of the sandwich structure constituting the binding of the target $\mathrm{IgG}$ molecule respectively to the GNP conjugated with rabbit antihuman IgG Fc fragment and GNP conjugated with goat antihuman IgG Fab fragment (Thermo Fisher Scientific, Rockford, Illinois) is shown in Fig. 1. When these two GNP antibody conjugates are mixed in dog blood serum (Veterinary Clinic, Purdue University) spiked with different concentrations of human $\mathrm{IgG}$, the binding of human IgG will induce GNP to form dimeric or high-order oligomeric aggregates through a typical antibody-antigenantibody sandwich structure that can then be evaluated for detection sensitivity using their respective diffusion times. While our goal in this work is not to evaluate the number of particles in the aggregates, we expect that a significant change in diffusion time could be noted on aggregation and used as a quantitative measure in clinical assay development.

All measurements were performed using a scanning confocal time-resolved microscopy with a 465-nm (40-MHz pulsing) picosecond laser as an excitation source (Picoquant, Germany). The details of the instrumentation can be found elsewhere. ${ }^{10}$ The autocorrelation curve of GNP diffusion was fitted to a 3-D diffusion model by Origin Lab as Eq. (1).

$$
G(\tau)=\frac{1}{\langle N\rangle}\left(1+\frac{\tau}{\tau_{D}}\right)^{-1}\left(1+\frac{\tau}{\tau_{D} \cdot \kappa^{2}}\right)^{-1 / 2}
$$

Calibration of the confocal volume for autocorrelation studies was performed with rhodamine 123 before actual measurements. The autocorrelation traces were fitted using the known diffusion coefficient $(D)$ of rhodamine (rho) 123 as $300 \mu \mathrm{m}^{2} / \mathrm{sec}$. The radial and axial beam were calculated by the expression $D=\omega^{2} / 4 \tau_{D}$, where $\omega$ is the radial beam size and $\tau_{D}$ is the diffusion time.

Next, 40-nm gold nanoparticles (GNPs) were synthesized by a citrate reduction of $\mathrm{HAuCl}_{4}{ }^{11}$ and functionalized with the respective biomolecules. Its diffusion characteristics in

1083-3668/2009/14(4)/040501/3/\$25.00 @ 2009 SPIE 

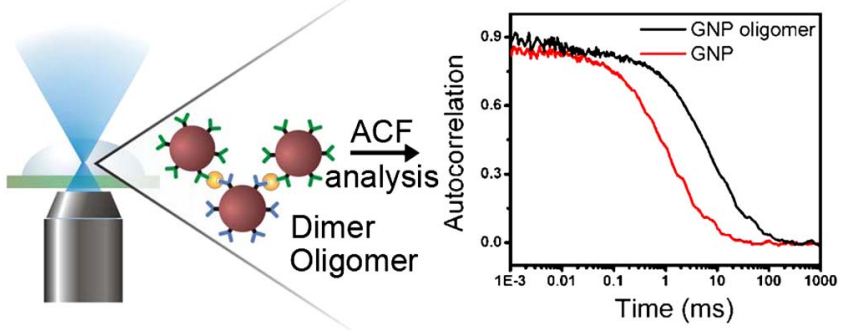

Fig. 1 Illustration of protein detection using GNR antibody conjugates by fluorescence correlation spectroscopy. ACF is autocorrelation function.

$20 \%$ dog blood serum were investigated. As shown in the count rate trace of GNPs from FCS, GNPs did not display any bleaching of their photoluminescence [Fig. 2(c)], which is a major advantage compared to conventional fluorescence dyes. The autocorrelation curve could be fitted well by a single component, indicating isotropic diffusion. The diffusion time of GNP obtained from Eq. (1) was 1.8 ms. Viscosity of $20 \%$ blood serum was determined by evaluating the change in diffusion coefficient of rho 123 from water to $20 \%$ blood serum. In addition to diffusion, the size of the particles was also back-calculated to confirm the estimations according to the Stokes-Einstein equation $D=K T /(6 \pi \eta d)$, where $k$ is the Boltzmann constant, $T$ is the temperature, and $\eta$ is the viscosity of liquid. From the calculations, the average hydrodynamic diameter of the diffusing GNP was estimated to be $42 \mathrm{~nm}$, which is in agreement with the size of the as-prepared 40-nm-diam GNP.

Before proceeding with immunoassay experiments, two batches of the citrate-coated GNP were separately conjugated with either the rabbit or goat antihuman IgG antibody fragment. To ensure attachment, ${ }^{5}$ biofunctionalization was assessed by the red shift of the surface plasmon resonance band for the GNP and GNP-antibody conjugates from the vis-NIR spectra (Jasco, Easton, Maryland) (data not shown). The antibody conjugation resulted in an increase in the diffusion time of GNP from 1.8 to $2.4 \mathrm{~ms}$, indicating an increase in the mean hydrodynamic size from 42 to $56 \mathrm{~nm}$ according to the Einstein-Stokes equation. The concentration of 40-nm GNP was estimated using a molar extinction coefficient of 7.666 $\times 10^{9} \mathrm{M}^{-1} \mathrm{~cm}^{-1}$ at a plasmon resonance wavelength maximum $\lambda_{\max }$ of $528 \mathrm{~nm}^{3}$

Since the single-component diffusion mode [Eq. (1)] is not accurate enough for estimating diffusion of antigen-induced GNP aggregations in a heterogeneous system, a multiplecomponent model with a maximum entropy method called MEMFCS $^{12}$ was used to obtain an optimal fit to further assess the autocorrelation. By introducing Eq. (1), $G(\tau)$ can be related to a continuous distribution of diffusion times in MEMFCS as follows:

$$
G(\tau)=\int \alpha_{i}\left(1+\frac{\tau}{\tau_{D}}\right)^{-1}\left(1+\kappa^{2} \frac{\tau}{\tau_{D}}\right)^{-0.5} d \tau_{D}
$$

The algorithm attempts to minimize the normalized chisquare value $\left(\chi^{2}\right)$ while maximizing the entropy $(S)$ term defined as $s=\sum_{i} p_{i} I n p_{i}$, where $p_{i}=\alpha_{i} / \sum \alpha_{i}$. Unlike the conventional fitting method where fitting components (i.e., 1,2,3, etc.) need to be set a priori, MEMFCS instead yields a size distribution that is discrete and particularly suitable for analysis of hetergeneous systems. The detection of human $\mathrm{IgG}$ in $20 \%$ dog serum was then demonstrated using the antibodyconjugated GNP based on FCS in the following experiments. The two gold nanoparticle probes (20 $\mu \mathrm{l}$ of $0.2-\mathrm{nM}$ concentration each) were added to $20 \mu \mathrm{l}$ of $20 \%$ dog serum, and the diffusion time was observed for varying concentrations $(0$, $10 \mathrm{ng} / \mathrm{mL}, 1 \mathrm{ng} / \mathrm{mL}, 0.1 \mathrm{ng} / \mathrm{mL}, 10 \mathrm{pg} / \mathrm{mL}, 5 \mathrm{pg} / \mathrm{mL}$, and $1 \mathrm{pg} / \mathrm{mL}$ ) of human IgG. $30-\mu \mathrm{L}$ solutions were used for measurement each time after incubating the GNP probes for $20 \mathrm{~min}$ at $37^{\circ} \mathrm{C}$. As expected, the half-value decay time of the normalized autocorrelation curves, which allows for a crude estimation of the diffusion time of the fluorescencing particles, varies significantly with increased antigen concentration [Fig. 2(a)]. The diffusion time distribution obtained using the MEMFCS fitting routine is shown in Fig. 2(b). The GNP probes show two predominant peaks with a higher IgG concentration $(10 \mathrm{ng} / \mathrm{ml})$, one at $2.5 \mathrm{~ms}$ and the other at
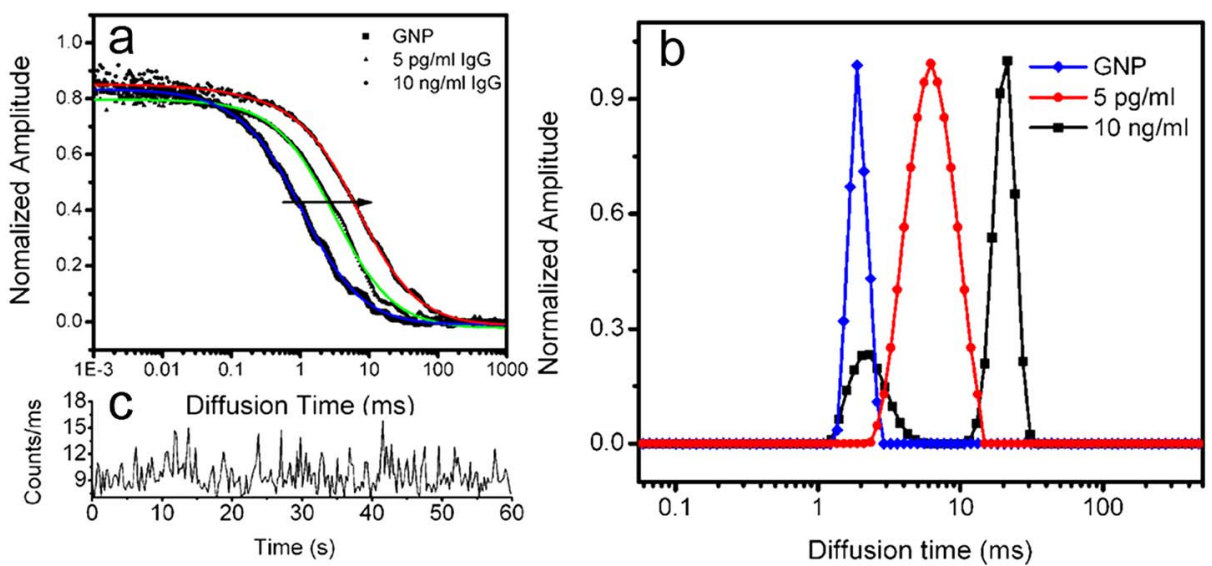

Fig. 2 (a) Autocorrelation spectra of $40 \mathrm{~nm}$ GNP ( $\mathbf{\square})$ and antigen-induced GNP oligomers in the presence of different concentrations of human IgG $[10 \mathrm{ng} / \mathrm{ml}(\mathbf{O}), 5 \mathrm{pg} / \mathrm{ml}(\mathbf{\Delta})]$ in $20 \%$ blood serum and the corresponding fitting curves (solid line). Arrow indicates increased diffusion time. (b) The diffusion time distribution of GNP with respect to antigen concentration and the resulting GNP oligomers from the MEMFCS fit. (c) Count-rate trace of GNPs with average size of $40 \mathrm{~nm}$ during FCS measurements. 


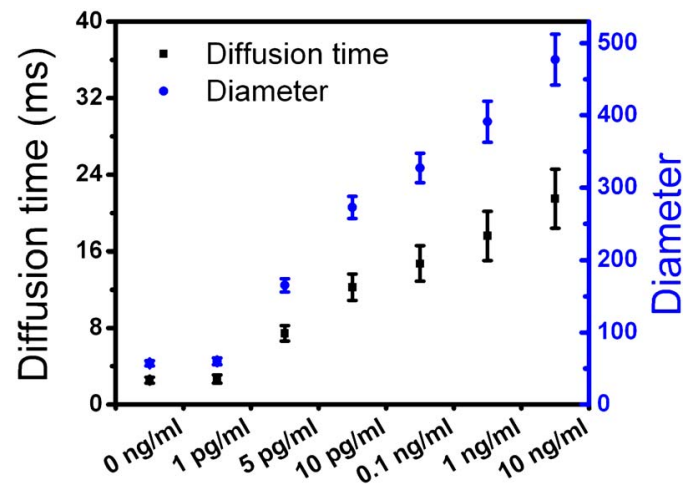

Fig. 3 Diffusion time and corresponding hydrodynamic diameter distribution of different concentrations of human $\operatorname{lgG}(0,1 \mathrm{pg} / \mathrm{ml}$, $5 \mathrm{pg} / \mathrm{ml}, 10 \mathrm{pg} / \mathrm{ml}, 0.1 \mathrm{ng} / \mathrm{ml}, 1 \mathrm{ng} / \mathrm{ml}$, and $10 \mathrm{ng} / \mathrm{ml}$ ) induced GNP probe aggregation plot as determined by FCS.

around $21 \mathrm{~ms}$, corresponding to free GNP and oligomeric GNP, respectively. However, bare GNP showed a single peak around $1.9 \mathrm{~ms}$, which is in a good agreement with the onecomponent fit $(1.8 \mathrm{~ms})$. GNP probes with $5-\mathrm{pg} / \mathrm{ml} \mathrm{IgG}$ concentration showed a peak at $7 \mathrm{~ms}$, which is in the range between 2 to $10 \mathrm{~ms}$, indicating the presence of both free and oligomeric GNP oligomers. Figure 3 shows the diffusion time and hydrodynamic diameter of GNP aggregates in the presence of different concentrations of human $\operatorname{IgG}$ in $20 \%$ blood serum. As the antigen concentration increased from 0 to $10 \mathrm{ng} / \mathrm{ml}$, the size of oligomers increased from 56 to $470 \mathrm{~nm}$, and the diffusion time of GNP aggregates increased accordingly with higher antigen concentration (2.5 to $21 \mathrm{~ms}$ ), as shown in Fig. 3. To further confirm the sandwich configuration resulting from the binding of the two GNP antibody conjugates ( $\mathrm{Fa}$ and $\mathrm{Fc}$ probes), specifically to the target (human $\mathrm{IgG}$ ), nonspecific binding control experiments were carried out with the two GNP probes simultaneously suspended in $20 \%$ serum without human $\operatorname{IgG}$ $(0 \mathrm{ng} / \mathrm{mL})$. In the control experiment, the diffusion time of the GNP probes was similar to the free GNP, demonstrating that the probes were stable with respect to nonspecific aggregation in blood serum, and their antigen-induced aggregation can be detected via the FCS at the single-particle level. Each measurement was replicated three times with three different sample preparations to demonstrate reproducibility to provide an assay at a LOD of $5 \mathrm{pg} / \mathrm{mL}$. To the best of our knowledge, this is the first blood serum immunoassay to report sensitivities in this order within $30 \mathrm{~min}$ at the single-particle level. It is also important to mention that the detection limit of our assay can be improved by orders of magnitude in buffers, because protein-rich environments of blood serum could hinder the assay performance, in addition to contributing to background scattering noise.

In conclusion, by taking advantage of the superior optical property of GNPs and single-molecule spectroscopy, a labelfree immunoassay that is simple, rapid, and ultrasensitive was shown to detect antigens at a LOD of $5 \mathrm{pg} / \mathrm{mL}$ in blood serum. Compared to traditional plate-based methods, our strategy is wash-free and can detect very low concentrations of targets directly in blood serum within minutes without.

\section{Acknowledgments}

Funding from the PRF and the CSFE-USDA grant is acknowledged. We thank Dr. Maiti and Periasamy (TIFR, Mumbai) for providing the MEMFCS software.

\section{References}

1. C. C. Lin, Y. M. Yang, Y. F. Chen, T. S. Yang, and H. C. Chang, "A new protein A assay based on Raman reporter labeled immunogold nanoparticles," Biosens. Bioelectron. 24(2), 178-183 (2008).

2. M. H. Chowdhury, V. A. Gant, A. Trache, A. Baldwin, G. A. Meininger, and G. L. Cote, "Use of surface-enhanced Raman spectroscopy for the detection of human integrins," J. Biomed. Opt. 11(2), 024004 (2006).

3. P. K. Jain, K. S. Lee, I. H. El-Sayed, and M. A. El-Sayed, "Calculated absorption and scattering properties of gold nanoparticles of different size, shape, and composition: applications in biological imaging and biomedicine," J. Phys. Chem. B 110(14), 7238-7248 (2006).

4. H. He, C. Xie, and J. Ren, "Nonbleaching fluorescence of gold nanoparticles and its applications in cancer cell imaging," Anal. Chem. 80(15), 5951-5957 (2008).

5. C. Wang, J. Chen, T. Talavage, and J. Irudayaraj, "Gold nanorod/ $\mathrm{Fe}(3) \mathrm{O}(4)$ nanoparticle nano-pearl-necklaces for simultaneous targeting, dual-mode imaging, and photothermal ablation of cancer cells," Angew. Chem., Int. Ed. Engl. 48(15), 2759-2763 (2009).

6. G. Malengo, A. Andolfo, N. Sidenius, E. Gratton, M. Zamai, and V. R. Caiolfa, "Fluorescence correlation spectroscopy and photon counting histogram on membrane proteins: functional dynamics of the glycosylphosphatidylinositol-anchored urokinase plasminogen activator receptor," J. Biomed. Opt. 13(3), 031215 (2008).

7. F. Fujii and M. Kinjo, "Detection of antigen protein by using fluorescence cross-correlation spectroscopy and quantum-dot-labeled antibodies," ChemBioChem 8(18), 2199-2203 (2007).

8. H. Wang, T. B. Huff, D. A. Zweifel, W. He, P. S. Low, A. Wei, and J. $\mathrm{X}$. Cheng, "In vitro and in vivo two-photon luminescence imaging of single gold nanorods," Proc. Natl. Acad. Sci. U.S.A. 102(44), 1575215756 (2005).

9. X. Liu, Q. Dai, L. Austin, J. Coutts, G. Knowles, J. Zou, H. Chen, and Q. Huo, "A one-step homogeneous immunoassay for cancer biomarker detection using gold nanoparticle probes coupled with dynamic light scattering," J. Am. Chem. Soc. 130(9), 2780-2782 (2008).

10. L. T. Varghese, R. K. Sinha, and J. Irudayaraj, "Study of binding and denaturation dynamics of IgG and anti-IgG using dual color fluorescence correlation spectroscopy," Anal. Chim. Acta 625(1), 103-109 (2008).

11. G. Frens, "Controlled nucleation for the regulation of the particle size in monodisperse gold suspensions," Nature (London), Phys. Sci. 241, 20-22 (1973).

12. P. Sengupta, K. Garai, J. Balaji, N. Periasamy, and S. Maiti, "Measuring size distribution in highly heterogeneous systems with fluorescence correlation spectroscopy," Biophys. J. 84(3), 1977-1984 (2003). 